\title{
An evaluation of the long-term complications associated with totally implantable venous access in oncology patients
}

\author{
Ahmet Acıpayam ${ }^{1}$, Erdinç Eroğlu² (D) \\ 'Department of Thoracic Surgery, Kahramanmaraş Sütçü İmam University Faculty of Medicine, Kahramanmaraş, Turkey \\ ${ }^{2}$ Department of Cardiovascular Surgery, Kahramanmaraş Sütçü Imam University Faculty of Medicine, Kahramanmaraş, Turkey
}

\begin{abstract}
Objectives: The aim of this study was to examine long-term complications developing in venous port catheters inserted in oncological patients.

Patients and methods: A total of 205 oncology patients (124 males, 81 females; mean age: $50.1 \pm 22.3$ years; range, 6 months to 90 years) in whom vascular port catheters were inserted between March 2015 and April 2020 were retrospectively analyzed. The first preference for port catheter application was the right internal jugular vein. If failed, the contralateral internal jugular vein was used. The procedure was carried out in the operating room with ultrasound (US) guidance under general anesthesia. All patients were evaluated in terms of pneumothorax, catheter orientation, and kinking and malposition after the procedure.
\end{abstract}

Results: A total 219 vascular ports were inserted in 205 patients. The indication for port catheter insertion was the receipt of long-term chemotherapy. Catheters were changed in four patients due to infection and in five patients due to catheter migration. The sites of catheter migration were the right atrium in two, the right ventricle in one, the main pulmonary artery in one, and the right pulmonary artery in one patient.

Conclusion: Port catheter insertion under US guidance is a well-designed procedure which can be performed with low complication rates by an experienced surgeon in an aseptic environment. Even if complications develop, they can be usually successfully treated in most cases.

Keywords: Chemotherapy, complication, port, ultrasound, venous access.

Implantable central venous port catheters are inserted into the large veins from the skin bed in a minor surgical procedure. They are used for long-term intravenous chemotherapy, antibiotic infusion, blood product transfusion, and repeated blood specimen collection. Venous port catheters are frequently used in oncology patients in particular to avoid peripheral venous route problems, if long-term chemotherapy is to be administered. ${ }^{[1,2]}$

The use of central venous access devices and port catheters, in particular, has increased in recent years due to the frequent venous access requirements in cancer patients, the long-term nature of treatment, the use of sclerosing agents, and the administration of large quantities of fluid. Their advantages include insertion under local anesthesia, minimal patient discomfort, low complication rates, and the fact that patients discharged on the same day can continue treatment at home. ${ }^{[3]}$ However, despite all their benefits for cancer patients, various complications may occur during the insertion or use of port catheters. Complications in the early period include pneumothorax, hemothorax, malposition, malfunction, arrhythmia, cardiac perforation, hematoma in the port pocket, embolism, arteriovenous fistula, left thoracic duct lesion, and phrenic or brachial plexus lesion. ${ }^{[4]}$ Skin necrosis, catheter breakage and embolism, infection, catheter

Received: September 01, 2020 Accepted: October 25, 2020 Published online: January 14, 2021

Correspondence: Erdinç Eroğlu, MD. Kahramanmaraş Sütçü İmam Üniversitesi Tip Fakültesi, Kalp ve Damar Cerrahisi Anabilim Dall, 46040 Onikişubat, Kahramanmaraş, Türkiye. e-mail: erdinc046@hotmail.com 
occlusion and disconnection, fluid extravasation, and difficulty in port location, and blood aspiration may be encountered in the late period. ${ }^{[3,4]}$

In the present study, we aimed to evaluate long-term complications developing in venous port catheters inserted in oncological patients.

\section{PATIENTS AND METHODS}

A total of 205 patients (124 males, 81 females; mean age: $50.1 \pm 22.3$ years; range, 6 months to 90 years) in whom vascular port catheters were inserted at Kahramanmaraş Sütçü Imam University, Faculty of Medicine, Departments of Throcic Surgery and Cardiovascular Surgery, between March 2015 and April 2020 were retrospectively analyzed. The principal indication for port catheter insertion was the long-term receipt of chemotherapy. Patients who had a port catheter in our clinic but whose treatments and follow-ups were performed in different hospitals were not included in the study. Patients were evaluated in terms of general living conditions, hemorrhagic diathesis, and the presence of any mass or local infection in the port catheter insertion site. A written informed consent was obtained from each patient and/or parent. The study protocol was approved by the Kahramanmaraş Sütçü Imam University, Faculty of Medicine, Ethics Committee (No. 12, Session: 2020.09, Date: 3.05.2020). The study was conducted in accordance with the principles of the Declaration of Helsinki.

The site of preference for port catheter application was the right internal jugular vein. However, in failed cases, the contralateral internal jugular was used. The jugular vessels were evaluated using ultrasound (US) examination prior to sterilization of the catheter region. The procedure was performed in the operating room under local anesthesia in adult patients and under general anesthesia in all pediatric cases. Catheters with a single lumen port were used for all patients (SecurePort ${ }^{\circledR}$, Amora, Italy; POLYSITE ${ }^{\circledR}$ 4008 ISP, Ivry-le-Temple, France; districAth ${ }^{\circledR}$, SaintEtienne, France). The subcutaneous port pocket was prepared in the anterior chest wall. Once the port body and catheter components were connected, the port reservoir was washed with $100 \mathrm{U} / \mathrm{mL}$ heparinized fluid and inserted in the subcutaneous pocket. Following the procedure, all patients were evaluated in terms of pneumothorax, hemothorax, kinking in the catheter, and catheter malpositioning complications using chest X-rays. Non-complicated patients were discharged $4 \mathrm{~h}$ after the procedure.
They were also prescribed oral antibiotics (ampicillin/ sulbactam $150 \mathrm{mg} / \mathrm{kg} / \mathrm{day}$ in three doses for children and ampicillin/sulbactam t.i.d. $g$ for adults) and analgesics (paracetamol $10 \mathrm{mg} / \mathrm{kg}$ q.i.d. for children and paracetamol $4 \times 500 \mathrm{mg}$ for adults). All patients were examined in terms of complications such as hematoma in the implantation site, erythema, edema, and suture separation in the postoperative first week.

\section{Surgical technique}

The procedures were performed in the operating room, under local anesthesia in adults and general anesthesia for children. Cardiac rhythm and arterial blood pressure were monitored, and venous puncture was performed with US guidance using an 18-gauge venous needle. Following venous puncture, a 0.035 -inch guidewire was inserted through the internal jugular vein. Next, a subcutaneous pocket was prepared by blind dissection of the subcutaneous tissue with an approximately 2 to $3-\mathrm{cm}$ skin incision beneath the clavicle to prepare the subcutaneous pocket to receive the port body. Particular care was taken to ensure that the pocket was of a suitable size for the port reservoir. A tunnel was, then, created between the port pocket and the catheterization region with the assistance of a trocar. A $9-\mathrm{Fr}$ port catheter in adults or a 6.5 -Fr port catheter in children was inserted through this tunnel. A separable sheath was installed over the guidewire, after which the guidewire was removed. The catheter was, then, cut to an appropriate length, with one end of the catheter being inserted through this sheath. The sheath was next separated and removed. The other end of the catheter was inserted into the port body. The connection between the catheter and port reservoir was checked for any leakage using a Huber ${ }^{\mathrm{TM}}$ needle. The port reservoir was, then, attached to the chest wall with two silk sutures, and the subcutaneous tissue and skin incision were closed using the subcuticular technique.

All discharged patients were followed at every outpatient control visit, until removal of the port catheter. The presence of hyperemia in the cutaneous port entry site or above the subcutaneous tunnel along which the port was inserted and the port catheter drum, or discharge in the incisions were evaluated. In case of ward hospitalizations, the port catheter was washed with physiological saline at every use and was closed with heparinized water.

\section{Statistical analysis}

Statistical analysis was performed using the PASW for Windows version 17.0 software (SPSS Inc., 
Chicago, IL, USA). Continuous variables were expressed in mean \pm standard deviation (SD) or median (min-max), while categorical variables were expressed in number and percentage. The Fisher's exact test was used to compare categorical variables between the groups. A $p$ value of $<0.05$ was considered statistically significant.

\section{RESULTS}

A total 219 vascular ports were inserted in 205 patients. The mean age was $6.5 \pm 5$ (range, 0 to 6 ) years in pediatric patients and $57.6 \pm 12.9$ (range, 21 to 90 ) years in adults. Overall mean duration of catheterization was $290.6 \pm 304.2$ (range, 3 to 1,450 ) days. Intravenous drug administration for long-term chemotherapy represented the main indication for subcutaneous catheter insertion. Baseline demographic and clinical characteristics of the patients with their diagnoses and catheter indications are summarized in Table 1.

Catheters were inserted in the right internal jugular vein in $90.4 \%$ (198) of cases, and in the left internal jugular vein in $9.6 \%$. Control posteroanterior $\mathrm{X}$-ray revealed that the catheter was inserted at a

Table 1. Demographic and clinical characteristics of patients $(n=205)$

\begin{tabular}{lcc}
\hline Variable & $\mathrm{n}$ & $\%$ \\
\hline Sex & & \\
Male & 124 & 60.5 \\
Female & 81 & 39.5 \\
Age (year) & & \\
$\quad<18$ & 30 & 14.6 \\
$\geq 18$ & 175 & 85.4 \\
Port implantation period (days) & & \\
$<300$ & 150 & 68.5 \\
$\geq 300$ & 69 & 31.5 \\
Anesthesia & & \\
Local & 182 & 83.1 \\
General & 37 & 16.9 \\
Port implantation site & & \\
Right internal jugular vein & 198 & 90.4 \\
Left internal jugular vein & 21 & 9.6 \\
Subclavian vein & 0 & 0 \\
Underlying disease & & \\
Malignant solid tumor & 186 & 90.7 \\
Breast cancer & $12^{*}$ & 5.9 \\
Gastrointestinal cancer & 139 & 67.8 \\
Gynecological cancer & 21 & 10.2 \\
Lung cancer & 5 & 2.4 \\
Other & 9 & 4.4 \\
Hematological malignancy & 19 & 9.3 \\
Metastatic cancer & 89 & 43.4 \\
\hline
\end{tabular}

narrow angle in the venous puncture site in one patient. This region was opened, and the catheter position was corrected. A minimal hematoma not obstructing the functioning of the port in the region of the port pocket developed in three patients. Port infection was suspected due to Candida growth in the cultures in two patients and due to uncontrollable fever in two patients, and the ports were removed from these patients. Opening in the port insertion site occurred in one patient. This area was debrided, and the port trunk was inserted more deeply.

Catheters were changed due to port catheter thrombosis in five patients and due to catheter fracture and migration in another five patients. The catheter migration site was the right atrium in two, the right ventricle in one, the main pulmonary artery in one, and the right pulmonary artery in one patient. In cases of migration, the femoral vein was entered in the hybrid operating room using percutaneous methods, and the catheter was removed with a loop-snare. No hemothorax, pneumothorax, or venous thrombosis developed in any patient. No deaths caused by central venous access device complications were recorded in our series over a period of five years (Table 2).

\section{DISCUSSION}

Ultrasound-guided totally implantable central venous port systems (TICVPS) which increase adherence to treatment and comfort in oncology patients can minimize rates of complications such as pneumothorax and hemothorax. No pneumothorax or hemothorax developed in any of the US-guided venous port systems inserted in our patients. Skin erosion around the port chamber developed in one patient during follow-up, catheter migration in five, catheter malfunction in one, thrombosis in the catheter in five,

Table 2. Complications of the totally implantable central venous port system

\begin{tabular}{lcc}
\hline & \multicolumn{2}{c}{ Frequency } \\
\cline { 2 - 3 } Type of complication & $\mathrm{n}$ & $\%$ \\
\hline Chamber site-associated & & \\
$\quad$ Infection & 1 & 5.26 \\
Skin erosion at the chamber insertion site & 1 & 5.26 \\
$\quad$ Hematoma & 3 & 15.79 \\
Catheter associated & & \\
Infection & 3 & 15.79 \\
Thrombosis & 5 & 26.32 \\
Migration & 5 & 26.32 \\
Malfunction & 1 & 5.26 \\
Total & 19 & 100.00 \\
\hline
\end{tabular}




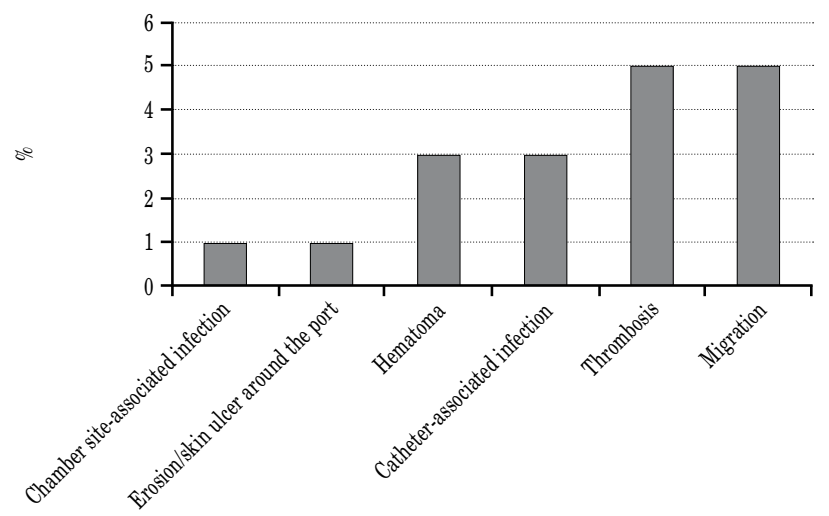

Figure 1. Distribution of complications.

and catheter infection in three patients (Figure 1). The port chamber in the patient with skin erosion continued to be employed by inserting it in a deeper position. The migrated catheters were removed using angiographic techniques in the hybrid operating room. The present study shows that TICVPS are well-designed tools that can significantly avoid puncture-related complications when performed in an aseptic environment, by an experienced team, and under US guidance. The results also indicate that complications that may emerge in the long-term can be easily treated in the presence of an experienced team.

The first use of the TICVPS was reported by Niederhuber et al. ${ }^{[5]}$ in 1982 . Since then, the system has become increasingly used in patients undergoing chemotherapy, parenteral nutrition, intravenous injection, transfusion or repetitive laboratory analysis. The TICVPS is applied to more than 15 million patients annually in the United States, and the rate of associated complications ranges between 5 and 19\%. ${ }^{[6]}$ The system is capable of lowering infection rates and thrombosis deriving from recurrent puncture of the veins in oncology patients. Also, it has no or only a minimal impact on recipients' daily lives, and the cosmetic results after implantation are usually good. The TICVPS allows a safe access to the central vein and long-term comfort and cosmetic satisfaction for patients requiring long-term venous access.

The fact that the right internal jugular vein and superior vena cava form a straight line has been reported to reduce catheter contact with the vascular wall, thus lowering the risk of venous thrombosis. ${ }^{[7]}$ Our preference was, therefore, to use the right internal jugular vein when possible for venous access during port catheter insertion. The left internal jugular vein was adopted as the

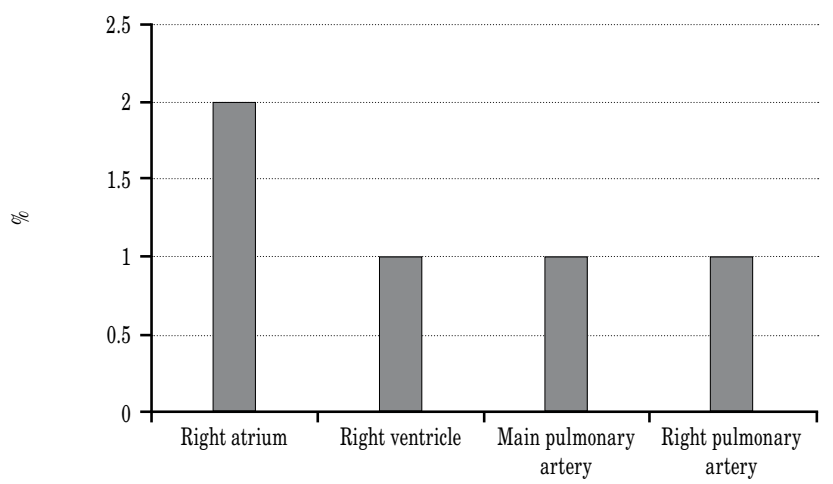

Figure 2. Distribution of patients developing catheter migration.

secondary access vein in the presence of an anatomical abnormality in the right internal jugular vein, or in patients with right breast cancer, undergoing radical axillary lymph node dissection and postoperative radiotherapy. ${ }^{[8]}$ Subclavian puncture should be avoided to obviate iatrogenic pneumohemothorax and pinch-off syndrome. Potential complications in the early period such as pneumothorax, air embolism or arterial puncture may be fatal, although none of these were observed in the present study. The US-guided puncture also lowers the rates of these complications.

In their study, Teichgräber et al. ${ }^{[9]}$ reported an infection rate of 0.8 to $7.5 \%$ in association with the port catheter use. No port infection was detected in the early period (within the first seven days) in the present study. All patients in this study underwent TICVPS in an operating room meeting the aseptic criteria for a standard surgical room and for imaging equipment, which may have helped to minimize sources of infection. The ports were removed due to port-related infection in four patients $(1.95 \%)$ in the subsequent period. Hyperemia was present in the port insertion pocket region in one case, while recurrent febrile episodes occurred after one month following the procedure in the remaining patients. The ports were removed due to Candida growth in the cultures in two patients, while no other source of infection could be identified in the other two patients, which were, therefore, regarded as port catheter infection. Our basic approach in cases of port infection involves the removal, if no response is achieved to medical treatment, followed by the insertion of a new catheter from the contralateral side.

One important complication of central venous catheters is catheter-related thromboembolism. 
Port catheter thrombosis usually occurs in the longterm, and increases in-hospital morbidity and reduces the quality of life of the patient. ${ }^{[10]}$ The estimated incidence of catheter-related thrombosis in cancer patients is between 3 and 40\%. ${ }^{[10]}$ Precautionary measures represent the first step in combating mechanical complications, such as port thrombosis and infection. Thus, a good port maintenance is particularly important. Care of the port catheter system in the present study involved washing with normal physiological saline and subsequent refilling with diluted heparin-physiological saline (300 $\mathrm{U}$ heparin inside $3 \mathrm{~mL}$ physiological saline). The port needles were replaced every seven days. No thrombosis was detected in our patients in the early period (within the first seven days). Catheter thrombosis developed in five (2.4\%) patients four to six months after the procedure, and these catheters were removed.

Insertion site complications related to the TICVPS include infection and erosion. Infection of the chamber insertion site may assume the form of erythema, tenderness, and occasional discharge. ${ }^{[11]}$ One patient with chamber insertion site infection in this study presented with redness, swelling and pain, and antibiotherapy was administered. However, no improvement was registered, and the port was removed and replaced by a new port catheter on the contralateral side.

Skin erosion occurring at the insertion site is an uncommon long-term complication. ${ }^{[12]}$ This typically involves the breakdown of the skin overlying the chamber, resulting in exposure of the device in the subcutaneous space. The process of skin erosion is a gradual one that leads to infection. Such infection may manifest systemically in the form of fever with chills and/or locally in the form of discharge or abscess. However, erosion with no infection has also been reported. ${ }^{[12]}$ Skin erosion can be also caused by incision site tension, repeated abrasion, or repeated needle puncture. An incidence of skin erosion of $1 \%$ was reported in a recent study. ${ }^{[13]}$ The incidence of skin erosion in the present study was $0.5 \%(1 / 205)$. The pocket was larger than the chamber (semicircular $\sim 2 \mathrm{~cm}$ in diameter) to reduce skin incision tension after insertion. We also created a thick skin flap designed to withstand repeated puncture and weight loss. Patients undergoing chemotherapy frequently lose weight as a result of chemotherapy-associated side effects, and a thick skin flap should, therefore, be employed in these cases.
Furthermore, the TICVPS has a good safety profile, although complications are not unknown. Late mechanical complications include catheter fracture and cardiac migration, although these are very rare, occurring in only 0.1 to $1 \%$ of cases. ${ }^{[14]}$ However, these may result in arrhythmia, thromboembolism, infection, and cardiac arrest, necessitating endovascular or surgical fragment removal. ${ }^{[15]}$ Various types of catheter material, particularly polyurethane, have been also linked to an increased risk of fracture. ${ }^{[16]}$ The improvement may be due to a better port catheter technology bestowing a greater resistance to shearing and higher-pressure infusion. ${ }^{[17]} \mathrm{A}$ fractured catheter fragment may migrate to various different sites, including the inferior-superior vena cava, right atrium, right ventricle, hepatic vein, left and right pulmonary artery, and coronary sinus, although the right heart chambers are the most common sites. ${ }^{[18]}$ Cather migration occurred in $2.4 \%$ $(5 / 205)$ of the patients in the present study. Two cases involved migration to the right atrium, one to the right ventricle, one to the main pulmonary artery, and one to the right pulmonary artery. These were removed using percutaneous methods, entering the femoral vein, in the hybrid operating room.

The clinical presentation of catheter fractures and migration may exhibit a wide spectrum, including being entirely asymptomatic, resistance to irrigation, mild pain or swelling in the clavicular region, shoulder pain, chest pain or discomfort, palpitation, and even lethal arrhythmias. ${ }^{[19]}$ However, the majority of presentations are benign, with patients reporting no or minimal symptoms. The increased resistance to infusion/irrigation was the most common indication of catheter fractures reported in study. ${ }^{[20]}$ Resistance to irrigation, mild pain or swelling in the clavicular region, or shoulder pain were observed in our patients.

While some authors have recommended that the TICVPS should be removed on completion of treatment, some others have advocated that the system can be left in situ, without any clear consensus on this subject. ${ }^{[21]}$ In our clinical practice, we prefer not removing the catheter at the end of treatment.

There are some limitations to the present study. First, despite a detailed analysis, the study has a retrospective and single-center design. Second, both pediatric and adult patients were included in the study and each population was unable to be evaluated separately. Another limitation is that a single brand of catheter was unable to be employed which may have resulted in variation in the material quality and may have, in turn, affected the complication rates. 
In conclusion, TICVPS insertion-related complication rates were low in this study. Complications can be, to a large extent, avoided with well-designed procedures, experienced vascular surgeons, an aseptic environment, and US-guided puncture. Even if complications occur in the long-term management of TICVPS, these can be usually treated without any difficulty. Long-term catheter survival rates are excellent, and catheter-related mortality rates are extremely low in oncology patients. Nonetheless, further well-designed, large-scale, long-term, prospective studies are needed to confirm these findings.

\section{Declaration of conflicting interests}

The authors declared no conflicts of interest with respect to the authorship and/or publication of this article.

\section{Funding}

The authors received no financial support for the research and/or authorship of this article.

\section{REFERENCES}

1. Biffi R, De Braud F, Orsi F, Pozzi S, Arnaldi P, Goldhirsch A, et al. A randomized, prospective trial of central venous ports connected to standard open-ended or Groshong catheters in adult oncology patients. Cancer 2001;92:1204-12.

2. Kutun S, Ulucanlar H, Ağaç A, Demir A, Çetin A. Non-complicated central venous port catheter among 21 years without examination: Case report. Turk J Vasc Surg 2007;16:31-4.

3. Ballarini $C$, Intra $M$, Pisani Ceretti A, Cordovana A, Pagani M, Farina G, et al. Complications of subcutaneous infusion port in the general oncology population. Oncology 1999;56:97-102.

4. Kim DH, Ryu DY, Jung HJ, Lee SS. Evaluation of complications of totally implantable central venous port system insertion. Exp Ther Med 2019;17:2013-8.

5. Niederhuber JE, Ensminger W, Gyves JW, Liepman M, Doan K, Cozzi E. Totally implanted venous and arterial access system to replace external catheters in cancer treatment. Surgery 1982;92:706-12.

6. McGee DC, Gould MK. Preventing complications of central venous catheterization. N Engl J Med 2003;348:1123-33.

7. Cimochowski GE, Worley E, Rutherford WE, Sartain J, Blondin J, Harter H. Superiority of the internal jugular over the subclavian access for temporary dialysis. Nephron 1990;54:154-61.
8. Araújo C, Silva JP, Antunes P, Fernandes JM, Dias C, Pereira $\mathrm{H}$, et al. A comparative study between two central veins for the introduction of totally implantable venous access devices in 1201 cancer patients. Eur J Surg Oncol 2008;34:222-6.

9. Teichgräber UK, Pfitzmann R, Hofmann HA. Central venous port systems as an integral part of chemotherapy. Dtsch Arztebl Int 2011;108:147-53.

10. Wiegering V, Schmid S, Andres O, Wirth C, Wiegering A, Meyer T, et al. Thrombosis as a complication of central venous access in pediatric patients with malignancies: a 5-year single-center experience. BMC Hematol 2014;14:18.

11. Bishop L, Dougherty L, Bodenham A, Mansi J, Crowe $\mathrm{P}$, Kibbler $\mathrm{C}$, et al. Guidelines on the insertion and management of central venous access devices in adults. Int J Lab Hematol 2007;29:261-78.

12. Almhanna K, Pelley RJ, Thomas Budd G, Davidson J, Moore HC. Subcutaneous implantable venous access device erosion through the skin in patients treated with antivascular endothelial growth factor therapy: a case series. Anticancer Drugs 2008;19:217-9.

13. Lee AY. Cancer and thromboembolic disease: pathogenic mechanisms. Cancer Treat Rev 2002;28:137-40.

14. Shah M, Patni S, Bagarahatta R. Spontaneous chemoport fracture and cardiac migration. Indian J Surg Oncol 2014;5:325-6.

15. Kim JE, Kim MK, Shim YK, Kim JT, Kim SM, Lee SY, et al. Percutaneous retrieval of an embolized central venous chemoport in a patient with colon cancer. Korean Circ J 2012;42:122-4.

16. Alzahrani K, Lejeune J, Lakhal W, Morel B, Cook AR, Braïk K, et al. Polyurethane versus silicone port a cath: What's going on at removal? J Pediatr Surg 2018;53:1417-9.

17. Zerati AE, Wolosker N, de Luccia N, Puech-Leão P. Cateteres venosos totalmente implantáveis: histórico, técnica de implante e complicações. J Vasc Bras 2017;16:128-39.

18. Pignataro BS, Nishinari K, Wolosker N, Bomfim GA. Fracture and migration into the coronary sinus of a totally implantable catheter introduced via the right internal jugular vein. BMJ Case Rep 2014;2014:bcr2014207276.

19. Tabatabaie O, Kasumova GG, Eskander MF, Critchlow JF, Tawa NE, Tseng JF. Totally Implantable Venous Access Devices: A Review of Complications and Management Strategies. Am J Clin Oncol 2017;40:94-105.

20. Cheng CC, Tsai TN, Yang CC, Han CL. Percutaneous retrieval of dislodged totally implantable central venous access system in 92 cases: experience in a single hospital. Eur J Radiol 2009;69:346-50.

21. Simon A, Graf N, Furtwängler R. Results of a multicentre survey evaluating clinical practice of port and Broviac management in paediatric oncology. Klin Padiatr 2013;225:145-51. 